\title{
Angiosarcoma hepático idiopático y metástasis cardíaca
}

\section{Idiopathic Hepatic Angiosarcoma and Heart Metastasis}

Pedro Rosales Torres, MD, ${ }^{1}$ Rafael Pila Pérez, MD, ${ }^{1}$ Rafael Pila Peláez, MD, ${ }^{1}$ Pedro León Acosta, MD, ${ }^{1}$ Yoanis Socarras Vidal, MD. ${ }^{1}$

1 Hospital provincial docente Manuel Ascunce Domenech, medicina interna. Camagüey, Cuba.

Fecha recibido: $22-06-15$

Fecha aceptado: 26-01-16

\begin{abstract}
Resumen
El angiosarcoma hepático es una neoplasia poco frecuente cuya incidencia se reporta en el $0,5 \%-2 \%$ de las tumoraciones hepáticas primarias. El diagnóstico es tardío cuando el órgano está comprometido y no es posible la terapéutica quirúrgica; la mayoría es de naturaleza idiopática (75\%) y las manifestaciones clínicas y radiológicas son inespecíficas. El diagnóstico es fundamentalmente por estudio histopatológico. Se presenta el caso de un paciente masculino de 34 años, sin antecedentes de interés, con un tiempo de evolución de su enfermedad de 4 meses, que se presentó con tumoración hepática, síntomas inespecíficos y alteraciones cardiovasculares que ocasionaron la muerte por taponamiento cardíaco. Se realizó necropsia y el estudio histopatológico mostró angiosarcoma hepático con metástasis cardíaca y pleural.
\end{abstract}

\section{Palabras clave}

Angiosarcoma hepático, tumores del hígado, metástasis cardíacas.

\begin{abstract}
Hepatic angiosarcoma is a rare malignancy whose incidence is reported to be from $0.5 \%$ to $2 \%$ of primary liver tumors. Late diagnosis is defined as diagnosis after the liver has been compromised and surgical therapy is no longer possible. Most hepatic angiosarcomas (75\%) are idiopathic, and clinical and radiological findings are nonspecific. Diagnosis is mainly by histopathology. We report the case of a 34-year-old male patient with no history of interest who had had a liver tumor for four months without specific symptoms. Cardiovascular disturbances led to death by cardiac failure. An autopsy and histopathology revealed liver angiosarcoma that had metastasized to the heart and pleural cavity.
\end{abstract}

Keywords

Liver angiosarcoma, liver tumors, cardiac metastases.

\section{INTRODUCCIÓN}

El angiosarcoma hepático (AH) es una neoplasia mesenquimal que representa el $0,2 \%-2 \%$ de todas las neoplasias primarias del hígado (1). Los $\mathrm{AH}$ son un tipo raro de sarcomas en los que las células malignas expresan las propiedades morfológicas y funcionales de las células endoteliales, y representan menos del 1\% del resto de los sarcomas (1). Debido a su relativa rareza, no existen series extensas en la literatura, siendo escasa la experiencia en estos tumores, y los criterios para su manejo son aproximativos. La edad pico de incidencia es entre 60 y 70 años, con límites de edad entre la segunda y octava décadas. La relación hombre-mujer es de 3:1, siendo raro en jóvenes y niños; en series de casos reportados en Gran Bretaña y Estados Unidos, la incidencia anual es de 0,14-0,25 casos por millón $(1,2)$. Es una enfermedad de diagnóstico difícil, por lo que la mayor parte de las veces se conoce posmor- 
tem, según reportes en series que alcanzan al 100\% de los enfermos $(1,3)$. En la mayoría de los casos se desconoce su causa, siendo idiopática en el $75 \%$, aunque existen casos secundarios de la exposición de cancerígenos, principalmente cloruro de vinilo, thorotrast, arsenicales, compuestos estrogénicos, esteroides anabólicos, ciclofosfamida y fenelzina; la enfermedad de Von Recklinhausen, la hemocromatosis y la cirrosis también pueden ocasionarlo (1-4). Se ha postulado la participación de diversos genes y diferentes mutaciones de los mismos en la genealogía de este tipo de tumores (5); también se ha constatado que la exposición a cloruro de vinilo provoca mutaciones en K-ras-2 y en la proteína $\mathrm{p} 53$, alteración habitualmente encontrada en sarcomas hepáticos (5). La inactivación del gen $\mathrm{p} 16$ mediante la metilación de su promotor, también desempeña un papel decisivo en la patogenia del $\mathrm{AH}$ (5).

El objetivo de este trabajo es presentar el caso de un paciente joven con AH idiopático con metástasis cardíaca, lo cual es extremadamente infrecuente en esta entidad.

\section{CASO CLÍNICO}

Paciente de 34 años de edad, masculino, mestizo, profesor de secundaria, sin antecedentes patológicos personales $\mathrm{ni}$ familiares de interés, no fumador, no ingestión de bebidas alcohólicas o contacto con sustancias tóxicas. Refiere que desde hace 3 meses comienza a presentar, en hipocondrio derecho, al principio simple molestia, pero con el decurso de los días se fue intensificando, fijo, persistente, acompañado de tinte subictérico en ambas esclera y piel, fiebre sin precisar sus características, la cual mejora con antipiréticos, astenia, anorexia marcada y pérdida de peso de aproximadamente $8 \mathrm{~kg}$ en los últimos meses. Al mes de este cuadro comienza a presentar palpitaciones muy frecuentes, fatiga $y$ cuadros sincopales frecuentes acompañados de disnea cada día más intensa; acude a un cardiólogo, quien le indicó ingreso en nuestra sala.

En el examen físico presentó: síndrome constitucional importante con marcada astenia, anorexia, fiebre de $39^{\circ} \mathrm{C}$, ictericia de piel y mucosas, palidez cutáneo mucosa importante con petequias y equimosis diseminadas por el tórax y otras regiones del cuerpo; adenopatías periféricas y edemas bilaterales en miembros inferiores que llegan por encima de ambas regiones maleolares. Aparato respiratorio: frecuencia respiratoria de $30 \mathrm{rpm}$, murmullo vesicular abolido en ambas bases. Aparato cardiovascular: tonos apagados, taquicárdicos y arrítmicos; tensión arterial 110/70 mmHg; frecuencia cardíaca central de $112 \mathrm{lpm}$. Abdomen: hepatomegalia de $10 \mathrm{~cm}$ de consistencia dura, dolorosa y nodular; esplenomegalia grado II de la clasificación de Boye. El resto del examen físico no mostró alteraciones.

\section{ESTUDIO ANALÍTICO}

$\mathrm{Hb} 8,5 \mathrm{~g} / \mathrm{L}$, Hto 0,26 vol\%, leucocitosis $12000 \times 10^{-9} / \mathrm{L}$ con fórmula diferencial normal, plaquetas $70000 \times 10^{-3} / \mathrm{L}$, velocidad de sedimentación globular de $88 \mathrm{~mm} / 1^{\text {era }} \mathrm{h}$; glucemia, creatinina, iones, función renal, amilasa pancreática, VDRL y VIH normales. Bilirrubina total $23 \mathrm{mmol} / \mathrm{L}$ (VN: $5-17 \mathrm{mmol} / \mathrm{L})$, bilirrubina indirecta $8 \mathrm{mmol} / \mathrm{L}$, bilirrubina directa $5 \mathrm{mmol} / \mathrm{L}(\mathrm{VN}$ : máximo $4 \mathrm{mmol} / \mathrm{L})$, transaminasa glutámica oxalacética $300 \mathrm{UI} / \mathrm{L}$ (VN: 5-30 U/L), transaminasa glutámica pirúvica $145 \mathrm{mmol} / \mathrm{L}(\mathrm{VN}: 5-30 \mathrm{mmol} / \mathrm{L})$, lactato deshidrogenasa $50 \mathrm{UI} / \mathrm{L}$ (VN: $130-300 \mathrm{mmol} / \mathrm{L}$ ), gamma glutamil transpeptidasa 80 UI (VN: 8-35 UI), fosfatasa alcalina $300 \mathrm{UI} / \mathrm{L}$ (VN: 40-100 UI/L), alfafetoproteína $10 \mathrm{ng} / \mathrm{mL}$ (VN: $1-20 \mathrm{ng} / \mathrm{mL}$ ). Antígeno de superficie para virus de la hepatitis $\mathrm{B}$ y $\mathrm{C}$ no reactivos; tiempo de protrombina C 14 P 26; hierro sérico $5 \mathrm{mmol} / \mathrm{L}$ (VN: 14-28 mmol/L); conteo de reticulocitos, coagulación y sangrado normales. Proteínas totales en suero $50 \mathrm{~g} / \mathrm{L}$ (VN: 60-80 g/L). Radiografía de tórax: derrame pleural bibasal de mediana cuantía con aumento de volumen del área cardíaca a expensas de ambas cavidades. ECG: bloqueo de rama izquierda del haz de His, taquiarritmia por fibrilación auricular. Ultrasonido abdominal: aumento difuso del hígado de aspecto irregular con nódulos diseminados que miden entre 5-25 mm, no presenta dilatación de los conductos biliares. Esplenomegalia de $135 \mathrm{~mm}$. Tomografía axial computarizada (TAC) de abdomen y tórax: derrame pleural bilateral y aumento de volumen de ambas aurículas y ventrículos con derrame pericárdico, el abdomen mostró las mismas características que el ultrasonido y además una pequeña cantidad de líquido en cavidad abdominal.

A los 7 días de su ingreso, preparándose para biopsia hepática dirigida por TAC, el paciente presenta taponamiento cardíaco que requirió pericardiocentesis, extrayéndose $1051 \mathrm{~mL}$ aproximadamente de un líquido francamente serohemático y fallece a las 2 horas. Se practica estudio necrópsico que informa la presencia de angiosarcoma hepático idiopático con metástasis fundamentalmente en corazón y pleura (figuras 1 a 7).

\section{DISCUSIÓN}

Los tumores más frecuentes descritos son los hepatocarcinomas y colangiocarcinomas, ya que los $\mathrm{AH}$ primarios son muy difíciles de diferenciar de otros tumores vasculares del hígado por técnicas imagenológicas $(1,2)$. Estos tumores son comunes con un origen multicéntrico en el hígado y también se pueden encontrar en el bazo y la médula ósea (3); este caso era multicéntrico y afectaba también el bazo. Su forma de presentación clínica incluye signos y síntomas relaciona- 


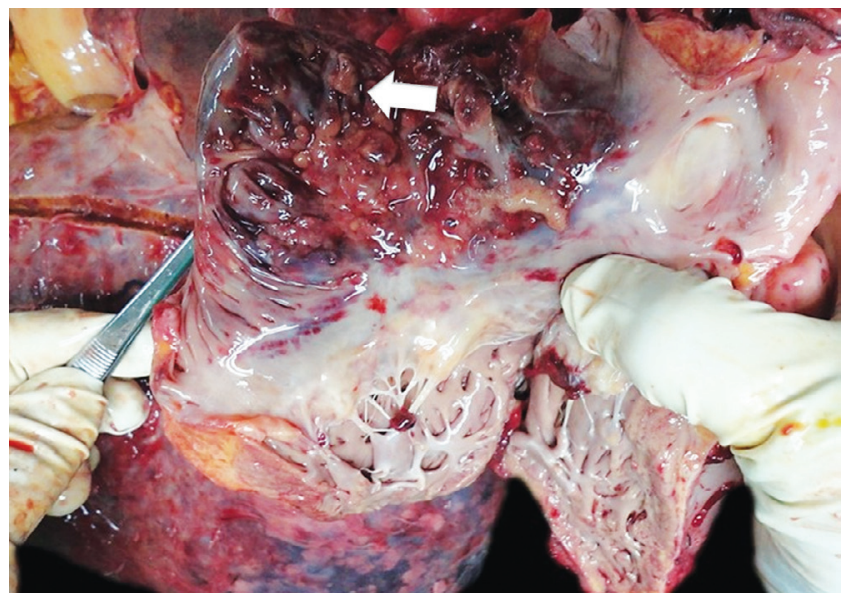

Figura 1. Macrofotografía de infiltración tumoral endomiocárdica con predominio de cavidades derechas.

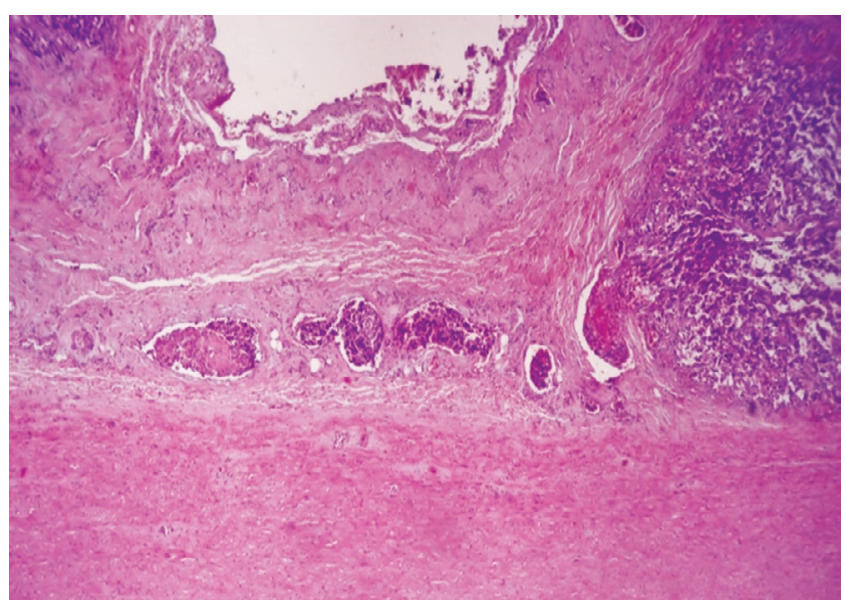

Figura 3. Corte histológico endomiocárdico. Obsérvese la infiltración metastásica en forma de nidos y grandes masas de células tumorales que dejan espacios claros de aspecto vascular (H/E-40 X).

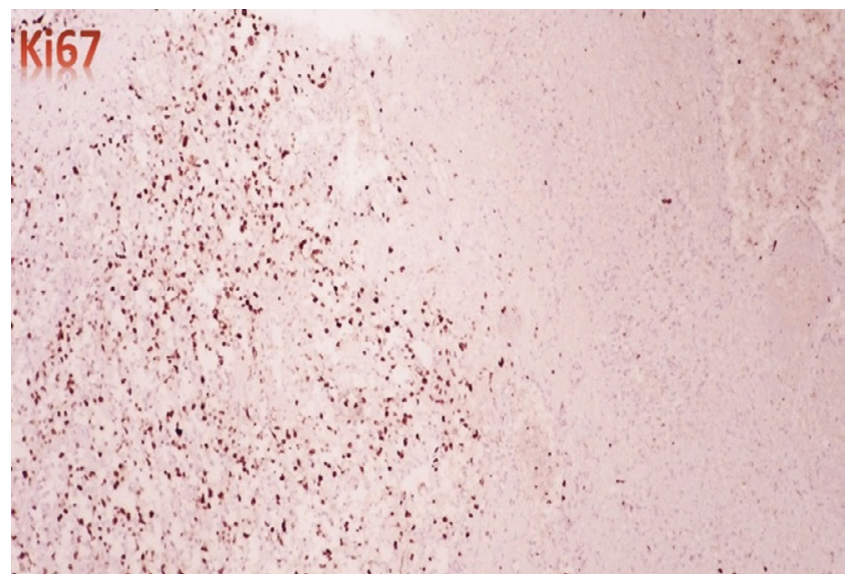

Figura 5. Imagen anterior. Obsérvese el marcado índice proliferativo en el área tumoral (IMQ-Ki 67 40X).

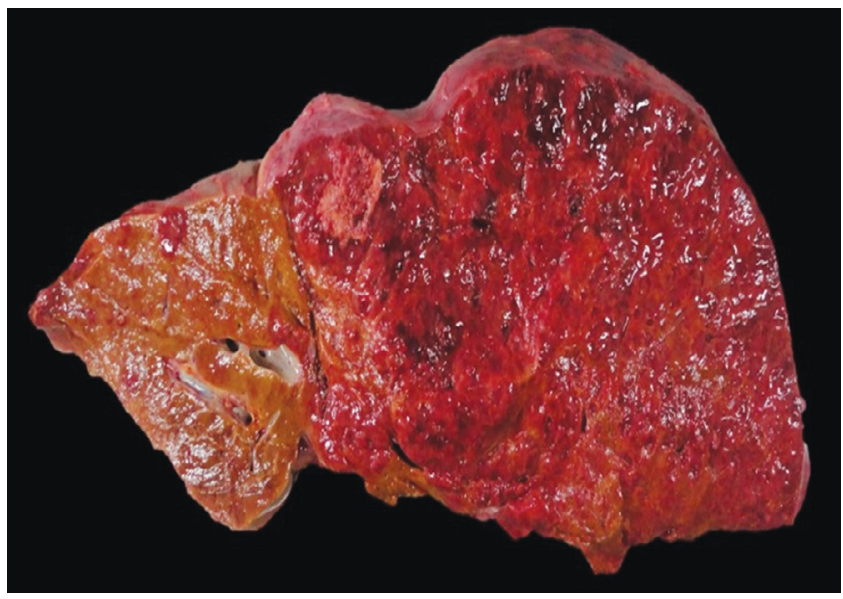

Figura 2. Corte frontal del lóbulo hepático. Se muestra gran tumoración abigarrada con zonas hemorrágicas que contrastan con porción de parénquima hepático normal a la izquierda de la foto.

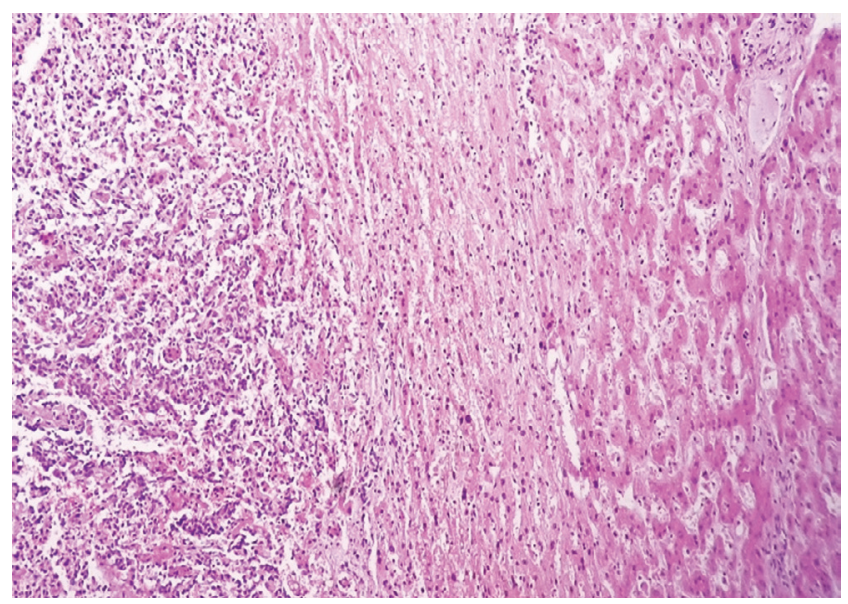

Figura 4. Microfotografía hepática. Infiltración tumoral (izquierda) que contrasta con parénquima hepático normal (derecha) (H/E-20X).

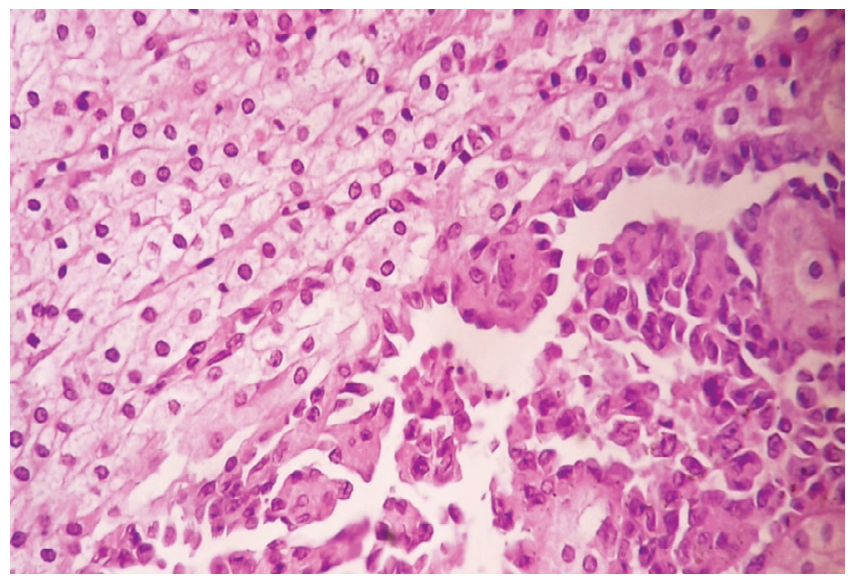

Figura 6. Imagen histológica de un campo tumoral. Obsérvese la proliferación de células endoteliales malignas típicas del angiosarcoma ( $\mathrm{H} / \mathrm{E}$ 40X). 


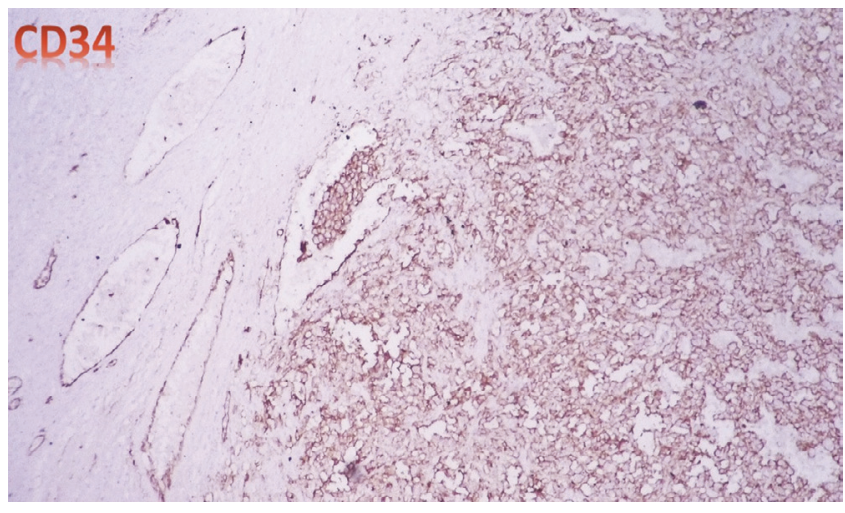

Figura 7. Microfotografía del área tumoral con técnica de inmunohistoquímica para vasos sanguíneos. Obsérvese la gran positividad en el área tumoral (IHQ-CD 34 positivo).

dos con enfermedad hepática como hepatomegalia, ascitis, dolor abdominal y pérdida de peso (62\%), todo ello constatado en este paciente. Puede presentarse con abdomen agudo por hemoperitoneo debido a la ruptura del tumor (15\%) y a la esplenomegalia (5\%), esta última reportada en este caso; igualmente, puede ocurrir fiebre de origen desconocido (1-5).

Las metástasis a distancia fundamentalmente se presentan en hueso, linfáticos, bazo y pulmón (9\%), pero en este enfermo fue llamativa la infiltración del corazón, la cual no está reportada, y de la pleura (1-4, 6). La insuficiencia hepática como consecuencia de la sustitución de los hepatocitos por células neoplásicas que lleva a necrosis secundaria, es rara $(3,5)$, pero se observó en este caso. Los parámetros analíticos no son específicos, más del $50 \%$ de los pacientes tiene trombocitopenia, alteraciones del perfil hepático con predominio de colestasis frente a citólisis y síndrome anémico ferropénico, que se consideran secundarias al secuestro que origina el tumor asociado con vascularidad; los marcadores tumorales son negativos (1-6). La trombocitopenia verdadera ocurre solo en individuos con hemoperitoneo masivo y consumo de plaquetas (2). Los estudios imagenológicos muestran diferentes patrones debido a la necrosis y la hemorragia (1-3, 7): la TAC muestra un tumor con múltiples masas hipodensas, estos hallazgos son similares a los hemangiomas (7); la resonancia magnética muestra la naturaleza hemorrágica, heterogénea e hipervascular de todas las masas dominantes (7).

El diagnóstico diferencial debe establecerse con sarcoma de Kaposi visceral, carcinoma hepatocelular hemorrágico, leiomiosarcoma vascular, hemangioendotelioma epitelioide maligno, procesos metástasicos difusos y peliosis hepática $(1-4,6)$; la cirrosis es el factor más importante en el diagnóstico diferencial radiológico, ya que más de $80 \%$ de los hepatocarcinomas se asocia con esta (1-4, 6, 7). Macroscópicamente, el tumor está formado por áreas grises que alternan con focos de hemorragias con grandes cavitaciones (1-4), observado en este paciente. En la mayor parte de los casos, el diagnóstico definitivo se basa en los resultados histopatológicos, en donde se encuentran grandes espacios cavernosos con proyecciones papilares hacia la luz, células tumorales, pleomórficas, hipercromáticas, ocasionalmente multinucleadas y con escaso citoplasma; lo ideal es la confirmación con inmunohistoquímica, con marcadores como CD-34 y CD-31 (8). En muchas ocasiones, las muestras obtenidas por punción con aguja fina (PAAF) no son concluyentes, por lo que siempre se recomienda una biopsia de hígado guiada por imagenología para evitar sangrados, ya que son tumores muy vascularizados (4).

El pronóstico es malo, la sobrevida global de los 5 años es de $35 \%$ (1-6), en casos localizados la sobrevida de los 5 años es de $60 \%$, con una media de supervivencia de 7 meses $(2,3)$; este enfermo tuvo una supervivencia desde el comienzo de los síntomas de entre 3 y 4 meses, con metástasis fundamentalmente en el corazón. El tratamiento para el AH es principalmente quirúrgico, pero debido al estadio avanzado en el momento del diagnóstico, tiene un papel limitado ya que podría mejorar las opciones de supervivencia en algunos casos; la laparoscopia debe ser la técnica quirúrgica de elección porque ofrece una visión directa de la cavidad abdominal y brinda información útil acerca del estadio y el pronóstico del tumor $(4,9)$. Muchos autores consideran que, en caso de enfermedad avanzada, ascitis o carcinomatosis peritoneal, la cirugía laparoscópica debe ser la técnica de elección. En general, se recomienda el uso de la radioterapia posresección en los sarcomas localizados de no gran tamaño, aunque se señala resistencia a la radiación (3). La indicación de trasplante hepático es controversial y la primera opción de tratamiento para el $\mathrm{AH}$ metástasico es la quimioterapia citotóxica, aunque la evidencia es no concluyente y los principales medicamento utilizados son las antraciclinas, las ifosfamidas y los taxanos (3, 7-10).

\section{CONCLUSIONES}

El angiosarcoma hepático es un tipo de cáncer de hígado que aparece en las células de Kupffer y endoteliales que revisten los vasos sanguíneos del hígado. Es una neoplasia extremadamente infrecuente y se considera idiopática por no mostrar posibles etiologías. Si no se extirpa por completo, la enfermedad es mortal al cabo de 3-6 meses. El pronóstico es poco alentador, dado que solo $6 \%-15 \%$ se puede extirpar con cirugía.

\section{Fuentes de financiación}

Los autores declaran no tener ningún apoyo económico externo. 


\section{Conflicto de intereses}

Los autores declaran no tener ningún conflicto de intereses.

\section{REFERENCIAS}

1. Flores Rivera OI, Quintana Quintana M, Frias Aguirre YN, González Cervantes JG, Baena Ocampo L. Angiosarcoma hepático: reporte de un caso y revisión bibliográfica. Med Int Mex. 2012;28(5):520-530.

2. Granado López SL, Gómez Jiménez LM, Chávez Bravo NC, Sánchez Rodríguez C. Angiosarcoma hepático idiopático. Informe de un caso. Rev Med Inst Mex Seguro Soc. 2012;50(4):445-448.

3. Young RJ, Brown NJ, Reed MW, Hughes D, Xxoll PJ. Angiosarcomas. Lancet Oncol. 2010;11:983-991.

4. Poggi Macituca L, Ibarra Chirinos O, López del Aguila J, Villanueva Pffucker M, Camacho Zacarías F, Tagle Arroslpide $\mathrm{M}$, et al. Angiosarcoma hepático: reporte de un caso y revisión de la literatura. Rev Gastroenterol Perú. 2012;32 (3):178-186.
5. Bhati CH S, Bhatt AN, Starkey G, Hubscher SG, Bramhatt SR. Acute liver failure due to primary angiosarcoma: A case report and review of literature. World J Surg Oncol. 2008;6:104-109.

6. Valenzuela J, Poveda MG. Angiosarcoma hepático. Presentación de dos casos. Rev Esp Enferm Dig. 2009; 101(6):430-437.

7. Koyama T, Fletcher JG, Johnson CD, Kuo MS, Notohara K, Burgart LJ. Primary Hepatic Angiosarcoma: findings at CT, med MR imaging. Radiology. 2002;222(3):6667-673.

8. Ying $\mathrm{CH}$. Liver Angiosarcoma a rare malignancy presented with intraabdominal bleeding due to ruptura: A case report. World J Surg Oncol. 2012;10:23-28.

9. Kim HR, RHA Sy, Cheon SH, Roh JK, Park YN, Yoo NC. Clinical features and treatment outcomes of advanced stage primary hepatic Angiosarcoma. An Oncol. 2009;20:780-787.

10. YAsunaka Y, Ikeda F, Kobashi H, Miyake Y, Takaki A, Iwasaki $\mathrm{T}$, et al. Hepatic Angiosarcoma with characteristic laparoscopy findings. Digest Endosc: Official Jpn J Gastroenterol Endosc Soc. 2012;24(2):124. 